\title{
Statistics of the slope-method estimator
}

\author{
Francesc Rocadenbosch, Adolfo Comerón, and Lorena Albiol
}

\begin{abstract}
The slope method has customarily been used and is still used for inversion of atmospheric optical parameters, extinction, and backscatter in homogeneous atmospheres from lidar returns. Our aim is to study the underlying statistics of the old slope method and ultimately to compare its inversion performance with that of the present-day nonlinear least-squares solution (the so-called exponential-curve fitting). The contents are twofold: First, an analytical study is conducted to characterize the bias and the mean-square-estimation error of the regression operator, which permits estimation of the optical parameters from the logarithm of the range-compensated lidar return. Second, universal plots for most short- and far-range tropospheric backscatter lidars are presented as a rule of thumb for obtaining the optimum regression interval length that yields unbiased estimates. As a result, the simple graphic basis of the slope method is still maintained, and its inversion performance improves up to that of the present-day computer-oriented exponential-curve fitting, which ends the controversy between these two algorithms. (C) 2000 Optical Society of America

OCIS codes: $\quad 010.0010,010.1290,010.3640$.
\end{abstract}

\section{Introduction}

The slope method first proposed by Collis ${ }^{1}$ has been used for more than three decades and is still used as a simple straightforward algorithm for estimating the atmospheric extinction and backscatter optical components in lidar systems under the assumption of a homogeneous atmosphere. This method departs from the simple single-scattering monostatic lidar equation $^{2}$

$$
P(R)=\frac{K}{R^{2}} \beta(R) \exp \left[-2 \int_{0}^{R} \alpha(r) \mathrm{d} r\right],
$$

where $P(R)$ represents the range-return power (in watts), $\beta(R)$ is the range-dependent atmospheric backscatter coefficient (in inverse kilometers per steradian), $\alpha(R)$ is the range-dependent atmospheric extinction coefficient (in inverse kilometers), $R$ is the range (in kilometers), and $K$ is the system constant (in watts times cubic kilometers) (a function of the laser energy and effective receiver area).

Under the assumption of a homogeneous atmo-

The authors are with the Department of Signal Theory and Communications, Antennas, Microwaves, Radar, and Optics Group, Universitat Politecnica de Catalunya, C/Sor Eulalia de Anzizu s/n, 08034 Barcelona, Spain. The e-mail address for F. Rocadenbosch is roca@tsc.upc.es.

Received 5 October 1999.

0003-6935/00/336049-09\$15.00/0

(C) 2000 Optical Society of America sphere $[\alpha(R) \approx \alpha, \beta(R) \approx \beta]$, the sought-after optical coefficients $(\alpha$ and $\beta$ ) can be estimated from slope $m$ and intercept $c$ of a linear regression applied to the logarithm of the range-corrected lidar signal:

$$
\begin{aligned}
S(R) & =\ln \left\{R^{2}[P(R)+n(R)]\right\} \\
& =\underbrace{m R+C}_{\text {ideal line }}+\frac{\ln \left[1+\frac{n(R)}{P(R)}\right]}{\text { noisy term }},
\end{aligned}
$$

with

$$
m=-2 \alpha, \quad c=\ln (K \beta) .
$$

The utility of the slope method increases with increasing optical depth, and, in particular, for optical depths greater than unity, a safe estimate can usually be obtained ${ }^{3}$ from Eq. (2) [if the signal-to-noise ratio (SNR) is high enough, the noisy term can be made redundant]. However, we must warn the reader that a priori information confirming the existence of a homogeneous atmosphere is strictly necessary. Otherwise, the algorithm may yield to situations in which an inhomogeneous reflection (e.g., a cloud reflection) cannot be distinguished from the returns of a homogeneous atmosphere (see Appendix B in Ref. 4). Other applications of the slope method in inhomogeneous atmospheres encompass boundary calibration of Klett's method ${ }^{3,5}$ and the so-called slice method of inversion, which is merely an extremely close variant of the slope method but applied to successive range intervals. ${ }^{6}$ 
As originally reported by Kunz and Leeuw, ${ }^{7}$ although the simplicity and straightforwardness of the slope method is advantageous, its biased estimation for low SNR's, caused by samples of the return signal approaching the noise floor $[n(R) \approx-P(R)$ in Eq. (2)], emerges as the main drawback of the algorithm. This effect is illustrated in Section 2, and in Section 3 we study the bias and the mean-square error (MSE) associated with the regression operator used in the slope-method algorithm from a statistical point of view.

Currently, with the computational tools available, direct application of the slope method itself has been superseded (in terms of inversion errors) by the application of nonlinear least-squares (NLSQ) procedures to exponential-curve fitting, as demonstrated in Ref. 8. Formally, we wish to solve

$$
\begin{aligned}
\min [\| S(R)-b & \left.\exp (-a R) \|^{2}\right]_{a, b}= \\
& \min \left\{\sum_{i=1}^{N}\left[S\left(R_{i}\right)-b \exp (-a R)\right]^{2}\right\}_{a, b},
\end{aligned}
$$

where $R_{i}$ is the range of the $i$ th observation cell and $a$ and $b$ are unbiased estimates of the terms $2 \alpha$ and $K \beta$, respectively.

The largest advantage of the exponential-curvefitting method over the customary slope method, in which all the available samples are considered for regression, is that the exponential fitting of Eq. (4) introduces no bias and therefore there is a lower inversion error result. This is because Eq. (4) gives more weight to the largest terms of the summation, which correspond to the first samples of $S(R)$ and for which the SNR is higher. However, its main disadvantages are that a computational approach is needed to solve nonlinear Eq. (4) by means of some numerical iterative recipe ${ }^{9}$ and that a reasonably good initialization is also needed for the algorithm to pay off. In such iterative solutions, having a good first guess, which often comes from the slope method as in the present case or from a look-up visibility table, is $90 \%$ of the battle; the inversion results are independent of the initialization method used, provided that the quality of the guess is good enough. Otherwise, unrealistic divergent results occur. ${ }^{7}$

In Section 4 we consider the optimum slope method, in which the optimum interval length for the minimum MSE is selected for regression, and its performance is compared with that of the NLSQ exponential-curve fitting introduced above [Eq. (4)]. Computation of the optimum length is presented in the form of universal plots suitable for most shortand far-range tropospheric backscatter lidars.

For the sake of simplicity in this study, we have assumed full overlap between the laser beam and the receiver's field of view in the regression interval of Eq. (2) and we have neglected the influence of the receiver bandwidth limitation on the estimated optical parameters. The former topic is covered in detail in Ref. 10, the latter in Ref. 11, and a practical rule for choosing the regression interval, given the range of

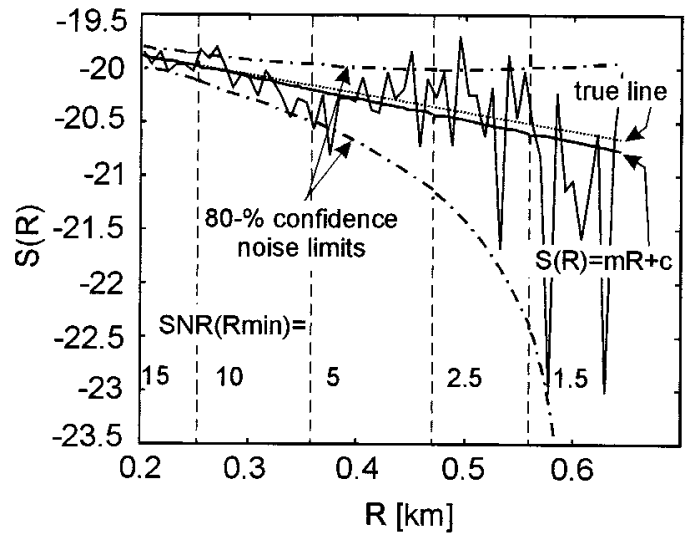

Fig. 1. Illustration of the bias effect for the slope method: solid curve, noisy realization of the log- and range-corrected signal $S(R)$ in Eq. (2); dotted line, unbiased ideal line built from the true atmospheric parameters in the simulator $\left(\alpha=1 \mathrm{~km}^{-1}, \beta=3 \times\right.$ $10^{-2} \mathrm{~km}^{-1} \mathrm{sr}^{-1}$ ); solid line, regressed line from the noisy $S(R)$ in application of the slope method. The line becomes tilted downward as a result of the estimator bias; dashed-dotted curves, $80 \%$ noise confidence limits for $N_{i}{ }^{\prime}$ [Eq. (8)].

full overlap and the receiver bandwidth, is given in Ref. 8.

\section{Noise Performance}

If the noise contribution $n(R)$ at each observation cell $R_{i}$ along the lidar exploration path is interpreted as a random variable $N_{i}$, then the range-corrected function $S(R)$ of Eq. (2) can be expressed as a set of random variables of the form

$$
S_{i}=\ln \left[R_{i}{ }^{2}\left(P_{i}+N_{i}\right)\right]=c+m R_{i}+N_{i}{ }^{\prime},
$$

where the log-noise and the observation-noise random variables $N_{i}{ }^{\prime}$ and $N_{i}$, respectively, are related by

$$
N_{i}^{\prime}=\ln \left(1+\frac{N_{i}}{P_{i}}\right)
$$

(Hereafter, capital letters represent random variables except for $P_{i}$ and $R_{i}$, which denote the deterministic return power and the range, already defined; lowercase letters represent feasible values of the random variable in its existence domain).

\section{A. Bias}

If the signal strength $P(R)$ is typically more than 50 photons over the integration time, as is always the case, the statistics of the observation-noise random variable $N_{i}$, which merges into single-body signalinduced dark-current shot noises and electronic thermal noise, can be assumed continuous Gaussian ones $^{8}$ (computation of the system's total noise variance is also given in the same reference). Consequently there is some likelihood, particularly higher for decreasing SNR's, that a noise realization of $N_{i}$ approaching the noise floor $-P_{i}$ at the $i$ th observation cell $R_{i}$ causes either a large noise spike in $N_{i}{ }^{\prime}$ or even a singularity in Eq. (6). This is illustrated in Fig. 1, in which it is evident that noise spikes at the end 

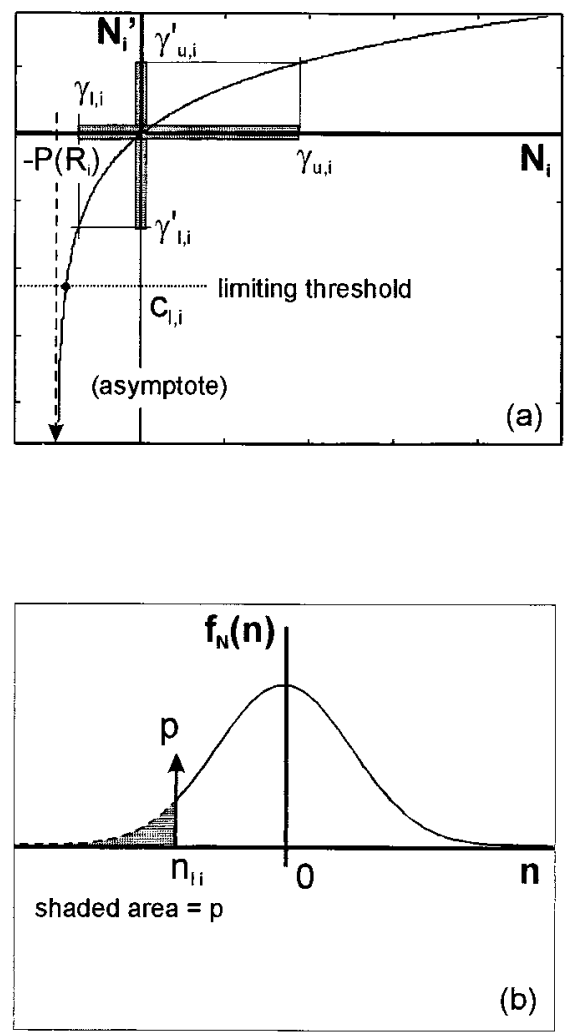

Fig. 2. (a) Equation (6) functional relationship between $N_{i}^{\prime}$ and $N_{i}$ random variables. Note that the symmetric interval $\left[\gamma_{l, i}, \gamma_{u, i}\right]$ for the Gaussian variable $N_{i}$ translates into an asymmetric one $\left[\gamma_{l, i}{ }^{\prime}, \gamma_{u, i}{ }^{\prime}\right]$ for variable $N_{i}{ }^{\prime}$. As a result $E\left[N_{i}{ }^{\prime}\right] \neq 0$ and the estimator becomes biased. (b) PDF of the threshold-limited Gaussian random variable $N_{i}$.

range are so large that the regressed line computed from direct application of the slope method [Eq. (2)] becomes biased (i.e., slightly tilted downward) from the ideal line built with the true atmospheric parameters $\left(\alpha=1 \mathrm{~km}^{-1}, \beta=3 \times 10^{-2} \mathrm{~km}^{-1} \mathrm{sr}^{-1}\right)$ in the simulator. ${ }^{8}$ This is how inversion errors arise.

The gist of the role bias plays on the slope method for different SNR's can be inferred from the limiting locus

$$
\left[S_{0}(R)-\gamma_{l}{ }^{\prime}(R), \quad S_{0}(R)+\gamma_{u}{ }^{\prime}(R)\right]
$$

in Fig. 1, where $S_{0}(R)$ is the noiseless ideal regression line indicated in Eq. (2) and $\gamma_{l}{ }^{\prime}(R)$ and $\gamma_{u}{ }^{\prime}(R)$ are two functions, still to be computed, that ensure at each range $R_{i}$ that

$$
p\left(N_{i}{ }^{\prime} \leq \gamma_{l, i}{ }^{\prime}\right)=10 \%, \quad p\left(N_{i}{ }^{\prime} \geq \gamma_{u, i}{ }^{\prime}\right)=10 \%,
$$

where the subscript $l$ indicates the lower limit, $u$ indicates the upper limit, and $i$ indicates $R_{i}$.

To solve Eq. (8) for $\gamma_{l, i}{ }^{\prime}$ and $\gamma_{u, i}{ }^{\prime}$, we use the fact that Eq. (6) is a monotonic increasing function [see Fig. 2(a)] and we use the basic properties of probability density function (PDF) transformations. ${ }^{12,13}$
By these means, the probability of $N_{i}{ }^{\prime}$ can be related to that of the Gaussian random variable $N_{i}$ as

$$
p\left(N_{i}{ }^{\prime} \leq n_{i}{ }^{\prime}\right)=p\left(N_{i} \leq n_{i}\right)=\frac{1}{2}+\frac{1}{2} \operatorname{erf}\left(\frac{n_{i}}{\sqrt{2} \sigma_{i}}\right),
$$

so that solution of the first equation of Eqs. (8) becomes

$$
p\left(N_{i}{ }^{\prime} \leq \gamma_{l, i}{ }^{\prime}\right)=0.1 \Rightarrow \gamma_{l, i}{ }^{\prime}=\ln \left[1-\frac{1.28 \sigma\left(R_{i}\right)}{P\left(R_{i}\right)}\right] .
$$

The procedure is analogous for $\gamma_{u, i}{ }^{\prime}$.

In addition, by using Eq. (8) and relation (10), we can establish a one-to-one relation between the $80 \%$ probability intervals in $N_{i}$ and $N_{i}{ }^{\prime}$ as follows:

$$
\begin{aligned}
p\left(\gamma_{l, i}{ }^{\prime} \leq N_{i}{ }^{\prime} \leq\right. & \left.\gamma_{u, i}{ }^{\prime}\right)=80 \% \\
& p\left(-1.28 \sigma_{i} \leq N_{i} \leq 1.28 \sigma_{i}\right)=80 \% .
\end{aligned}
$$

Finally, after introducing the SNR definition, we can write the solutions of Eqs. (8) as

$$
\gamma_{l, i}{ }^{\prime}=\ln \left[1-\frac{1.28}{\operatorname{SNR}\left(R_{i}\right)}\right], \quad \gamma_{u, i}{ }^{\prime}=\ln \left[1+\frac{1.28}{\operatorname{SNR}\left(R_{i}\right)}\right]
$$

Figure 1 shows the Eq. (7) locus for $\alpha=1 \mathrm{~km}^{-1}$, $R_{\text {min }}=0.2 \mathrm{~km}$, and $\operatorname{SNR}\left(R_{\text {min }}\right)=15$ (in linear units). Because for a given backscatter lidar system we can always relate the product $K \beta$ in Eq. (1) [or, alternatively, $K$ if the pair $(\alpha, \beta)$ is related by means of some look-up visibility table ${ }^{14,15}$ to the SNR at the starting point $R_{\min }$ of full overlap between the laser beam and the telescope field of view 7,8 [hereafter $\left.\operatorname{SNR}\left(R_{\text {min }}\right)\right]$, we can indistinctly parameterize our plots in terms of $\operatorname{SNR}\left(R_{\min }\right)$ 's or $K$ 's. Selection of $\operatorname{SNR}\left(R_{\text {min }}\right)$ instead of $K$ in Fig. 1 is preferred because the former is not related to any particular set of system parameters.

For $\operatorname{SNR}(R)<5$, a simple visual inspection of Fig. 1 is enough to reveal that the $N_{i}{ }^{\prime} 80 \%$ confidence limit starts to distribute asymmetrically. In other words, relation (11) translates a symmetric probability distribution (a Gaussian one) for $N_{i}$ into a nonsymmetric one for the log-random variable $N_{i}{ }^{\prime}$. This effect is at the core of the slope-method algorithm, and it is responsible for unwanted biased estimation $\left(E\left[N_{i}{ }^{\prime}\right] \neq 0\right.$, where $E[$.$] is the expectancy operation).$

\section{B. Singularities}

In theory, noise values $n_{i}$ equal to or exceeding the asymptotic noise floor $-P\left(R_{i}\right)$ in Fig. 2(a) lead to undefined figures in the logarithm of the rangecompensated signal $S(R)$. In practice, however, these singularities are avoided by means of some limiting criterion. Two of the most commonly used criteria consist of either discarding or resetting negative peaks of $S(R)$ larger than some predefined threshold $v_{l}$. In the former case, discarded points are not used for regression and the observation points $R_{i}$ become a nonuniform set. The latter case yields a uniform set $R_{i}$, and it is equivalent to limiting the domain of the 
$N_{i} \operatorname{PDF} f_{N_{i}}\left(n_{i}\right)$ to $\left[-\left|n_{l, i}\right|, \infty\right)$ [see Fig. 2(b)]. Formally, after Eq. (6)

$$
S_{i}=v_{l} \Rightarrow n_{l, i}=P_{i}\left[\exp \left(c_{l, i}\right)-1\right]
$$

where

$$
c_{l, i}=v_{l}-c-m R_{i}=\ln \left(1+\frac{n_{l, i}}{P_{i}}\right) .
$$

Note that in Eq. (13), the same limiting threshold $v_{l}$ yields to different limits, $n_{l, i}$, one for each cell along the exploration path. A good proposal for $v_{l}$ is to locate it approximately one unit below the expected $S\left(R_{\text {max }}\right)$. Formally,

$$
v_{l}=S_{1}-2 \hat{\alpha}_{\max }\left(R_{\max }-R_{1}\right)-1,
$$

where $S_{1}$ is the first sample of $S(R)$ and $\hat{\alpha}_{\max }$ is a user estimation of the largest expected extinction. This ensures that the limiting threshold $v_{l}$ is well below the ideal regression line [Eq. (2)].

Of course, each decision rule (resetting or discarding) leads to different statistics for the truncated Gaussian $N_{i}$ and therefore a different bias performance should, in principle, be expected. However, extensive simulation has shown that either a rule or the $v_{l}$ figure plays a significant role in the final estimator performance only if $\operatorname{SNR}\left(R_{\text {min }}\right)<30$. Taking into account that, for most tropospheric backscatter lidars $\operatorname{SNR}\left(R_{\min }\right)$ is approximately between $10^{2}$ and $10^{4}$ for atmospheric extinctions in the $10^{-2}-10-\mathrm{km}^{-1}$ range, ${ }^{8}$ we can assume that the statistical results that are derived next are not restricted to any decision rule, even though the resetting decision rule of Eq. (13) is assumed for simplicity reasons.

\section{Statistics of the Regression Operator}

Close inspection of Fig. 1 suggests that if we removed the far-end samples from the regression interval, bias would be considerably reduced because it is precisely the end part of the regression interval where the $80 \%$ confidence noise limits are most asymmetric. However, if the regression interval were too short, fewer useful samples would be used and therefore the inversion error would have a larger variance. As a result, it seems sensible to guess that an optimum regression interval length for minimum MSE exists.

In this study, we commit to the statistics of slope estimate $\hat{m}$ [Eqs. (3)] only because the procedure is completely similar for the intercept estimate $\hat{c}$, although mathematically it is much more involved and does not shed any new light on the concluding results.

\section{A. Bias and Mean-Square Error}

The estimates of slope $\hat{m}$ and intercept $\hat{c}$ for a straight-line fit to the log- and the range-corrected lidar set of data $\left\{x_{i}=R_{i}, y_{i}=S_{i}\right\}, i=1 \ldots N$ takes the form ${ }^{16}$

$$
\hat{m}=\frac{\overline{x y}-\overline{x y}}{\overline{x^{2}}-\bar{x}^{2}}, \quad \hat{c}=\bar{y}-\hat{m} \bar{x},
$$

where $\hat{m}$ and $\hat{c}$ yield the sought-after atmospheric optical estimates after Eqs. (3) and $x_{N}=R_{N}$ is defined as the maximum detection range; equivalently, $N$ represents the maximum number of available samples up to that range where the received power equals the noise-equivalent power of the receiver $\left[\operatorname{SNR}\left(R_{N}\right)\right.$ $=1]$. A plot of $R_{\max } \operatorname{versus} \operatorname{SNR}\left(R_{\text {min }}\right)$ is shown in Fig. 1 of Ref. 8, and a mathematical approximation of $R_{\text {max }}$ can be found in Appendix A of Ref. 7 .

Because for each incoming measurement, Eqs. (16) yield the pair of estimates $\hat{m}$ and $\hat{c}$, which are themselves random variables and are expected to be close to their atmospheric counterparts $m$ and $c$, we can compute the bias and the MSE associated with them. The estimator bias associated with $\hat{m}$ is defined as

$$
\operatorname{bias}=E[\hat{m}-m]=E[\hat{m}]-m,
$$

and the MSE takes the form

$$
\varepsilon_{\hat{m}}^{2}=E\left[(\hat{m}-m)^{2}\right]=E\left[\hat{m}^{2}\right]-2 m E[\hat{m}]+m^{2} .
$$

The solutions of Eqs. (17) and (18) require computation of the first and the second moments of $\hat{m}$ as follows.

We can easily compute the first moment of $\hat{m}$ in two steps, first by applying the expectancy operator to both terms of Eqs. (16) and second by expressing the expectancy of $S_{i}, E\left[S_{i}\right]$ as a function of the log-noise expectancy $E\left[N_{i}{ }^{\prime}\right]$ by means of Eq. (5). This yields

$$
E[\hat{m}]=m+\frac{1}{N Q} \sum_{i=1}^{N}\left(R_{i}-\bar{R}\right) E\left[N_{i}^{\prime}\right],
$$

where $Q$ will be defined by Eq. (25) for contextual reasons. The computation of $E\left[\hat{m}^{2}\right]$ is more involved, but it follows a similar procedure that finally reduces to the computation of $E\left[N_{i}{ }^{\prime}\right], E\left[N_{j}{ }^{\prime}\right]$. Assuming independence if $i \neq j$, we obtain

$$
\begin{aligned}
E\left[\hat{m}^{2}\right]= & E[\hat{m}]^{2}+\frac{1}{N^{2} Q^{2}} \sum_{i=1}^{N}\left(R_{i}-\bar{R}\right)^{2}\left(E\left[{N_{i}}^{\prime}{ }^{2}\right]\right. \\
& \left.-E\left[N_{i}{ }^{\prime}\right]^{2}\right) .
\end{aligned}
$$

Finally, substitution of Eq. (19) into Eq. (17) and of Eqs. (19) and (20) into Eq. (18) yields the sought-after bias and MSE, respectively. It follows that

$$
\operatorname{bias}=\frac{1}{N Q} \sum_{i=1}^{N}\left(R_{i}-\bar{R}\right) E\left[N_{i}{ }^{\prime}\right],
$$

$$
\varepsilon_{\hat{m}}^{2}=\frac{1}{N^{2} Q^{2}}(A+B),
$$


where

$$
\begin{aligned}
A & =\left(\sum_{i=1}^{N}\left(R_{i}-\bar{R}\right) E\left[N_{i}{ }^{\prime}\right]\right)^{2}, \\
B & =\sum_{i=1}^{N}\left(R_{i}-\bar{R}\right)^{2}\left(E\left[{N_{i}}^{\prime}{ }^{\prime}\right]-E^{2}\left[N_{i}{ }^{\prime}\right]\right) \\
& =\sum_{i=1}^{N}\left(R_{i}-\bar{R}\right)^{2}{\sigma_{N_{i}}{ }^{\prime}}^{2}, \\
Q & =\frac{1}{N} \sum_{i=1}^{N} R_{i}{ }^{2}-\bar{R}^{2} .
\end{aligned}
$$

Interpretation of the bias results of Eq. (21) becomes evident after our discussion in Subsection 2.A and identification of the term $E\left[N_{i}{ }^{\prime}\right]$.

For the MSE results of Eq. (22), it follows that the MSE is proportional to the sum of two terms, $A$ and $B$, which are related to different noise characteristics. The former depends on the estimation bias $E\left[N_{i}{ }^{\prime}\right]$, whereas the latter has to do with the log-noise power (i.e., the variance of $N_{i}{ }^{\prime}$ ). However, mean and variance of the $N_{i}{ }^{\prime}$ are not the only key factors that determine the final MSE performance because the noise contribution from each cell to the final MSE is weighted by the distance from that cell to the center of the regression interval $\left(R_{i}-\bar{R}\right)$. As a result, noise from cells located at the inversion interval boundaries is magnified. The definition of $Q$ in Eq. (25) is also related to the interval length, and it represents the variance of the discrete set $R_{i}$.

\section{B. Computation of the Optimum Regression Interval Length}

It is now time to justify our qualitative reasoning given in the introduction of Section 3 from a mathematical point of view by using Eqs. (22)-(25) in this way: Assume that we consider a subinterval of length $k<N$ samples, which begins at $R_{\min }$ as above so that we substitute $k$ by $N$ in Eqs. (22)-(25). If we increase the interval length $k$ toward $N$, which is equivalent to including new samples from the righthand side of Fig. 1 to the regression process, this not only increases $Q$ but also $A$ and $B$ terms in Eqs. (23) and (24), respectively. This is so because the new samples represent not only additional terms in the series but higher bias and variance contributions, which in turn are magnified by the fact that these samples are located farther from the interval center point $\bar{R}$. As a result, Eq. (22) becomes the ratio of two numbers progressively larger. As long as the increment of $Q$ offsets that of the term $A+B$, the MSE decreases and adding new samples to the regression interval is advantageous. However, for some $k \geq N_{\text {opt }}$ (note that $N_{\text {opt }}=N$ is also possible for large SNR's) this trend reverses and the MSE worsens. Consequently, we are interested in solving

$$
\frac{\mathrm{d} \varepsilon_{\hat{m}}^{2}}{\mathrm{~d} N}=0 \Rightarrow N=N_{\mathrm{opt}}
$$

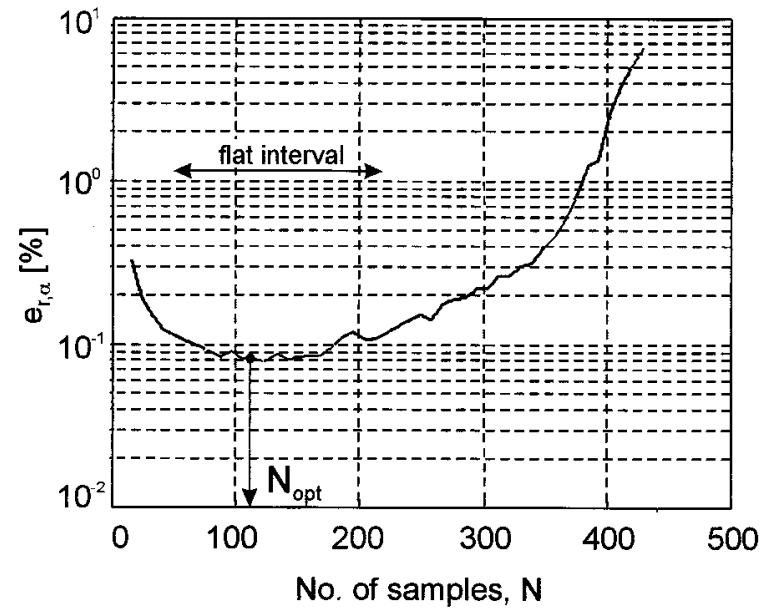

Fig. 3. Equation (22) plot of the relative inversion error [as defined in Eq. (27)] versus $N$. The study parameters are $\alpha=1$ $\mathrm{km}^{-1}, \mathrm{SNR}\left(R_{\min }=100, R_{\text {min }}=0.2 \mathrm{~km}, \Delta R=7.5 \mathrm{~m}\right.$ (sampling rate $20 \times 10^{6}$ samples/s), $\rho=2 \times 10^{-5} \mathrm{~Hz}^{-1 / 2}$.

The best way to do this is to find the minimum MSE from a MSE versus $N$ plot of Eq. (22), as shown in Fig. 3. Note that Fig. 3 plots the relative inversion error defined as

$$
e_{r, \alpha}=\frac{\sqrt{\mathrm{MSE}_{\hat{m}}}}{2 \alpha} \times 100 \% .
$$

The Fig. 3 plot requires successive evaluation of Eqs. (22)-(25) and, particularly, computation of the firstand the second-order moments of $N_{i}{ }^{\prime}, E\left[N_{i}{ }^{\prime}\right]$, and $E\left[{N_{i}}^{\prime 2}\right]$. In the most general case, such computation implies evaluation of the integral expressions in Eqs. (A5) and (A6) in Appendix A, but for $\operatorname{SNR}\left(N_{\text {opt }}\right)>9$ it has been found by experiment that approximations (B7) and (B8) are good, easy-to-use approximations that yield \pm 1 sample errors for most extinction ranges $\left(\alpha=0.1-10 \mathrm{~km}^{-1}\right)$. In general, for those interested in either of these two mathematical procedures (Appendix A or Appendix B), we advise rounding the $N_{\text {opt }}$ figure upward because the flat zone of the MSE versus $N$ plot always tend to be larger to the right of $N_{\text {opt }}$, as in Fig. 3. The procedure is relatively simple if the SNR enables us to use the approximated expressions given in Appendix B.

\section{Optimum Slope Method and Exponential-Curve Fitting}

The main problem with the procedure just described to solve Eq. (26) is that the whole computational procedure must be repeated for each particular case study, which comprises the following parameters: the (simulated) atmospheric extinction $\alpha$ under study, the minimum system range $R_{\text {min }}$, the spatial sampling $\Delta R$ (equivalently, the system acquisition rate), the $\mathrm{SNR}$ at the minimum range $\operatorname{SNR}\left(R_{\text {min }}\right)$, and, finally, a set of different system noise parameters, namely, the signal-induced and the darkcurrent shot-noise spectral densities and the thermal-noise spectral density $\sigma_{\mathrm{sh} \_s, i}{ }^{2}, \sigma_{\mathrm{sh} \_d, i}{ }^{2}$, and 
Table 1. Parameter Sets Used in the Compilation of the Universal Plots

\begin{tabular}{lc}
\hline$\alpha\left(\mathrm{km}^{-1}\right)$ & $0.1,1,10$ \\
$\operatorname{SNR}\left(R_{\min }\right)$ & $30,100,251,630,1584,3961,10.000$ \\
$R_{\min }(\mathrm{km})$ & $0.2,0.6,1$ \\
$\Delta R(\mathrm{~m})$ & $7.5,15,35,50$ \\
$\rho\left(\mathrm{Hz}^{-1 / 2}\right)[$ Eq. $(28)]$ & $7 \times 10^{-5}, 2 \times 10^{-5}, 6 \times 10^{-6}$ \\
\hline
\end{tabular}

$\sigma_{\mathrm{th}, i}{ }^{2}$, respectively. ${ }^{17,18}$ With so many parameters, the computation of Eq. (22) becomes cumbersome and time-consuming.

Working on the basis of simplicity has suggested to us the goal of drawing universal plots to solve $N_{\text {opt }}$. These plots should be parameterized by a subset of independent parameters so that the remaining parameters from those given above should play a secondary role in the determination of $N_{\text {opt }}$. The universality of these plots stems from the fact that we have considered typical high-, medium-, and lowperformance front-end lidar receivers, either avalanche photodiode or $\mathrm{p}$-i-n-based receivers.

The struggle to find the most appropriate subsets has led us to an advanced computer-oriented statistical study that solves Eqs. (22)-(26) for all combinations of the typical system parameters of Table 1 . For $\operatorname{SNR}\left(R_{\text {min }}\right) \geq 100$ in Table 1, approximations (B7) and (B8) have been used, but for $\operatorname{SNR}\left(R_{\text {min }}\right)=30$ we have had to resort to the integral computation of Eqs. (A5) and (A6). In addition, decorrelation studies have suggested the definition of an auxiliary noise parameter $\rho$, which merges into a single-body detector and system-noise parameters as follows:

$$
\rho=\frac{2 q F M}{\left(\sigma_{\text {sh_d }, i}{ }^{2}+\sigma_{\text {th }, i}^{2}\right)^{1 / 2}}\left(H z^{-1 / 2}\right),
$$

where $q$ is the electron change, $M$ is the avalanche photodiode multiplication factor, $F$ is its related excess-noise factor, ${ }^{19} \sigma_{\mathrm{th}, i}{ }^{2}$ is the system's equivalent thermal-noise spectral density (in square amperes per hertz) and $\sigma_{\mathrm{sh} \_d, i}{ }^{2}$ is the receiver's dark-current spectral density (in square amperes per hertz), both of which are referred to as the transimpedance amplifier's input (see Appendix A of Ref. 8 for further insight). [One can easily extend Eq. (28) to include p-i-n-based receivers by taking $F=1, M=1$ in Eq. (28).]

As a first result of the statistical simulation, we found that all the study cases of the Table 1 output curves are similar in shape to those in Fig. 3, all of which consist of a plateau-like interval around $N_{\text {opt }}$, which spans approximately $\pm 0.1 \mathrm{~N}$. In the second step, this morphological feature was put at the core of a regression study, as in the example of Fig. 4(a), which enabled us to identify $N_{\text {opt }} / N, \operatorname{SNR}\left(R_{\text {min }}\right)$, and the atmospheric extinction $\alpha$ as the primary subset and $R_{\min }, \Delta R$, and $\rho$ as the secondary subset. In all cases, the quality of the regressed data has been checked with the exact computation of $N_{\text {opt }}$, again by use of Eqs. (22)-(25). The relative regression error $\varepsilon_{\text {fit }}{ }^{\alpha}$ is typically less than $5 \%$ for $\alpha=10 \mathrm{~km}^{-1}$ [see the Fig. 4(b) histogram] and $\sim 1 \%$ for $\alpha=0.1-1 \mathrm{~km}^{-1}$ (not shown).

The universal plots, which enable us to solve the ratio $N_{\text {opt }} / N$ from an available estimation of the atmospheric extinction $\alpha$ (its order of magnitude) and $\operatorname{SNR}\left(R_{\text {min }}\right)$, are shown in Fig. 5 . Note that $N$ is defined as that range in which the received power is equivalent to the noise-equivalent power of the receiver $\left[\operatorname{SNR}\left(R_{N}\right)=1\right]$ and hence it must be known to solve $N_{\text {opt }}$ from the ratio $N_{\text {opt }} / N$.

An additional comment with regard to Fig. 5 is the asymptotic behavior of $N_{\text {opt }}$ with increasing $\operatorname{SNR}\left(R_{\min }\right)$. Thus, as long as $\operatorname{SNR}\left(R_{\text {min }}\right)$ increases, for each atmospheric extinction $\alpha$ and system parameter subset $\left(R_{\text {min }}, \Delta R, \rho\right), N_{\text {opt }}$ tends toward a saturation figure, which we determined as $\operatorname{SNR}\left(R_{\text {min }}\right)=$ 500 for $\alpha=10 \mathrm{~km}^{-1}, \operatorname{SNR}\left(R_{\text {min }}\right)=1500$ for $\alpha=1$ $\mathrm{km}^{-1}$, and $\operatorname{SNR}\left(R_{\min }\right)=10^{4}$ for $\alpha=0.1 \mathrm{~km}^{-1}$. Therefore there is no need to recompute $N_{\text {opt }}$ for $\operatorname{SNR}\left(R_{\text {min }}\right)$ 's greater than these SNR limits.

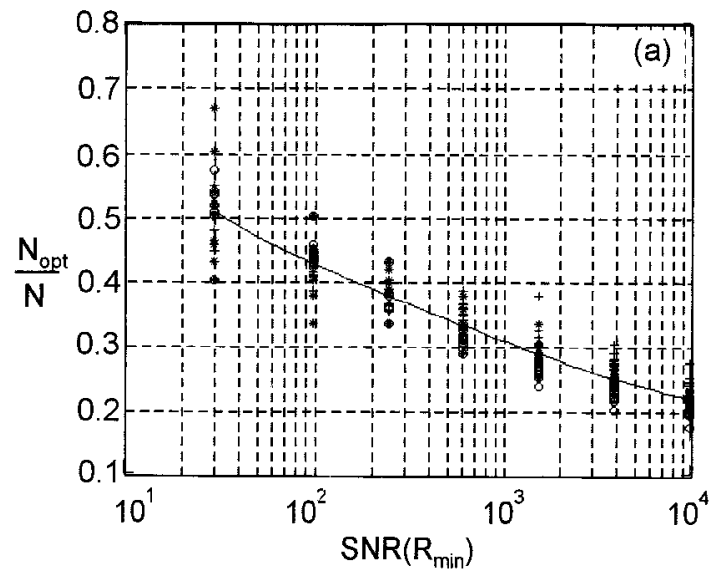

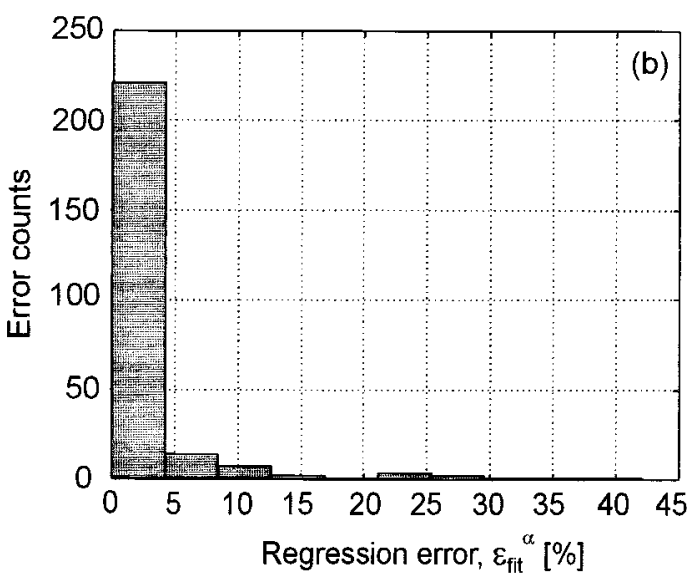

Fig. 4. (a) Example of a regression plot used to compute the universal plots. This plot solves $N_{\text {opt }} / N$ given $\operatorname{SNR}\left(R_{\text {min }}\right)$ for a simulated atmospheric extinction $\left(\alpha=10 \mathrm{~km}^{-1}\right.$ here). In this example, the regressed data sweep only $\rho$ from Table $1: \quad+, \rho=6 \times 10^{-6} \mathrm{~Hz}^{-1 / 2}$; $*, \rho=2 \times 10^{-5} \mathrm{~Hz}^{-1 / 2}$; and $\bigcirc, \rho=7 \times 10^{-5} \mathrm{~Hz}^{-1 / 2}$. Note that for most of the trials $N_{\text {opt }} / N$ is within a \pm 0.1 interval, which can be fitted into the flat zone of Fig. 3. (b) Binned data used to estimate the regression error quality, which is typically $\sim 5 \%$ for $\alpha=10 \mathrm{~km}^{-1}$. 


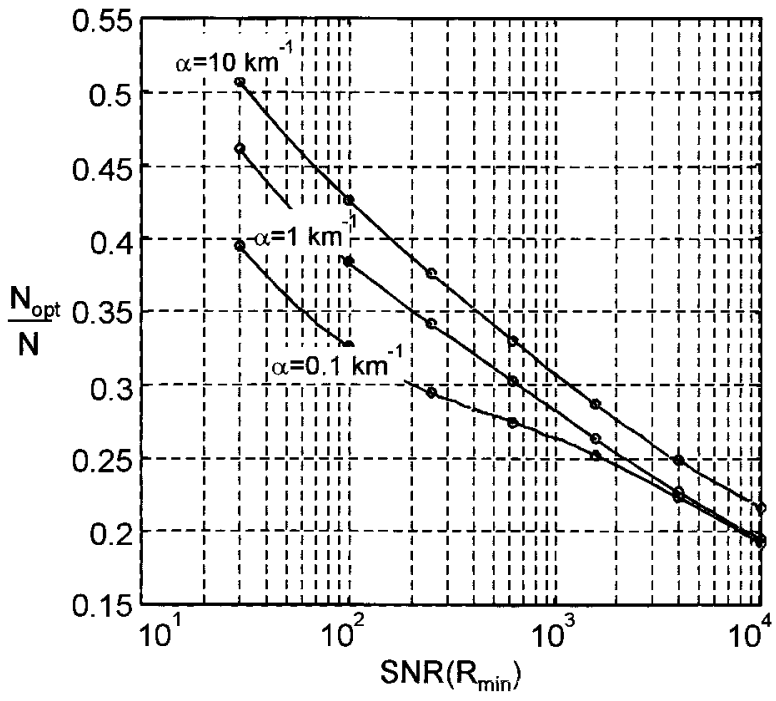

Fig. 5. Universal plots to solve $N_{\text {opt }}$ for minimum MSE in the slope method given $\operatorname{SNR}\left(R_{\text {min }}\right)$, an estimate of the order of magnitude of the atmospheric extinction $\alpha$, and $N$, which is defined as that range where the received power equals the noise-equivalent power of the receiver [i.e., $\operatorname{SNR}\left(R_{N}\right)=1$, as explained in Subsection 3.A].

Finally, Fig. 6 compares the error performances [Eq. (27)] achieved by the next three algorithms: Fig. 6(a), the customary slope method, i.e., by using all the available samples $N$ with $\operatorname{SNR}(R)>1$ for regression and by limiting $S(R)$ to a constant threshold $v_{l}$, as described in Subsection 2.B; Fig. 6(b), the optimum slope method discussed here, i.e., by using $N_{\text {opt }} \leq N$ samples for regression and the same limiting threshold as in Fig. 6(a) to cope with singularities; and Fig. 6(c), the exponential-curve fitting described in Section 1, Eq. (4), initialized from the results of the

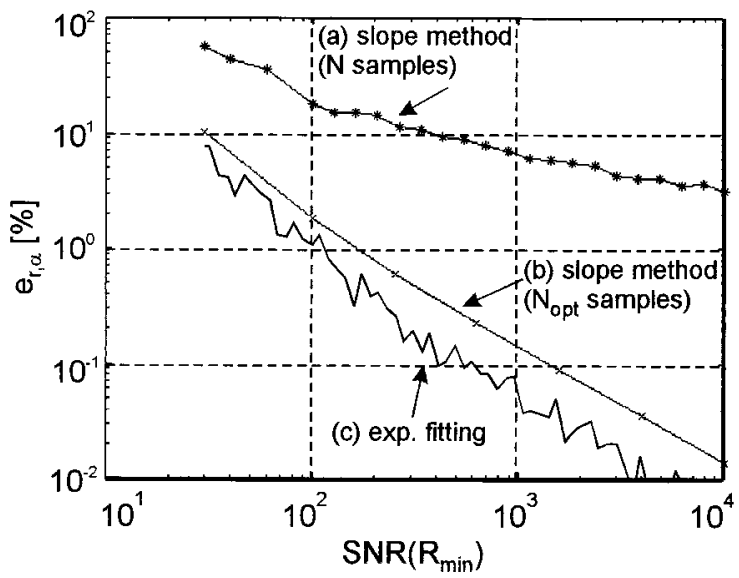

Fig. 6. Comparison of the relative inversion errors [Eq. (27)] among (a) the customary nonoptimized slope method, which uses all the available samples for inversion; (b) the optimized slope method, which uses the optimum interval length $N_{\text {opt }}$; (c) the NLSQ exponential fitting described in Section 1. It is shown that the performances of (b) and (c) algorithms unify in similar inversion errors, which ends an old controversy between the slope method and the exponential-curve fitting. slope method of Fig. 6(a) to prevent divergence of the NLSQ numerical routine. ${ }^{9}$

If we compare the plots in Figs. 6(a) and 6(b) and note the log scale in the grid, it becomes obvious that the most significant improvement with regard to the optimum slope method in lieu of the customary method is for moderate and poor $\operatorname{SNR}\left(R_{\text {min }}\right)$ 's. Thus, for low SNR's, such as $\operatorname{SNR}\left(R_{\text {min }}\right)=50, e_{r, \alpha}(N)$ $=40 \%$ and $e_{r, \alpha}\left(N_{\text {opt }}\right)=5 \%$ represent a $35 \%$ gain; for large $\operatorname{SNR}\left(R_{\text {min }}^{r, \alpha}\right)$ 's, such as $\operatorname{SNR}\left(R_{\text {min }}\right)=10^{4}, e_{r, \alpha}(N)$ $=3 \%$ and $e_{r, \alpha}\left(N_{\text {opt }}\right)=0.02 \%$ represent a $3 \%$ gain, although this is not significant in absolute terms. Similar conclusions follow for the rest of the atmospheric extinctions of Table 1.

Finally, a comparison of Figs. 6(b) and 6(c) proved that the slope method with optimum inversion length and the exponential-curve fitting converge to similar inversion error figures, with the optimum slope method being the simplest because the regression can even be performed graphically with the help of Fig. 5 . A comparison of Figs. 6(a) and 6(c) is also in good agreement with the error figures reported in Ref. 8. As a result, we can conclude that selection of the optimum regression interval length for the slope method yields a virtually unbiased estimator whose performance in a minimum MSE is similar to that of the exponential-curve fitting of Eq. (4).

\section{Conclusions}

A statistical and computer-oriented simulation study has been conducted to characterize the statistics of the well-known slope method, in particular, its bias and mean-square inversion error, and to compare its inversion performance with that of the exponentialcurve fitting by use of a previous study. ${ }^{8}$

It has been shown that the slope method is dominated by its inherent biased estimation and that inversion errors can largely be reduced if one chooses an optimum interval length for regression. To compute this length, analytical expressions, either exact or approximated, have been derived [Eqs. (22)-(25) plus Eqs. (A5) and (A6) for $\operatorname{SNR}\left(R_{\text {min }}\right)=30$ or approximations (B7) and (B8) for $\left.\operatorname{SNR}\left(R_{\text {min }}\right) \geq 100\right]$ as well as the quick-reference universal plots of Fig. 5, which are valid for most typical elastic-backscatter lidar systems according to Table 1.

Finally, we found that, when such an optimum regression length is considered, the MSE of the slope method reduces to that of the unbiased exponentialcurve-fitting estimator [Eq. (4)], and hence both algorithms are equivalent in terms of minimum bias and MSE performance.

\section{Appendix A: Integral Computation of $E\left[N_{i}{ }^{\prime}\right], E\left[N_{i}^{\prime 2}\right]$}

We depart from the Gaussian-limited PDF random variable $N_{i}, f_{N_{i}}\left(n_{i}\right)$, described in Subsection 2.B and depicted in Fig. 2(b) and from application of the expectancy theorem ${ }^{12}$ to Eq. (6), which states that the expectancy of a random variable $N_{i}{ }^{\prime}$ related to another random variable $N_{i}$ by means of a functional 
relationship [Eq. (6) in the present case] can be computed as

$$
E\left[N_{i}{ }^{\prime}\right]=\int_{-\infty}^{\infty} \ln \left(1+\frac{n}{P_{i}}\right) f_{N_{i}}(n) \mathrm{d} n .
$$

Based on the fact that the existence domain of $N_{i}$ is $\left[n_{l, i}, \infty\right)$ and that the interval that has been removed $\left(-\infty, n_{l, i}\right)$ is equivalent to a delta function with area (probability)

$$
p=\int_{-\infty}^{n n_{l, i}} \frac{1}{\sqrt{2 \pi} \sigma_{n, i}} \exp \left(-\frac{n^{2}}{2 \sigma_{n, i}^{2}}\right) \mathrm{d} n=\frac{1}{2} \operatorname{erf}\left(\frac{-n_{l, i}}{\sqrt{2} \sigma_{n, i}}\right),
$$

Eq. (A1) can be computed as

$$
\begin{aligned}
E\left[N_{i}{ }^{\prime}\right]= & \int_{-\infty}^{n_{l, i}} \ln \left(1+\frac{n}{P_{i}}\right) p \delta\left(n-n_{l, i}\right) \mathrm{d} n \\
& +\underbrace{\int_{n l, i}^{\infty} \ln \left(1+\frac{n}{P_{i}}\right) \frac{1}{\sqrt{2 \pi} \sigma_{n, i}} \exp \left[-\frac{1}{2}\left(\frac{n}{\sigma_{n, i}}\right)^{2}\right] \mathrm{d} n}_{\text {main term }} .
\end{aligned}
$$

After identifying $c_{l, i}$ [Eq. (14)] in the first integral term and introducing the following substitutions in the main term for enhanced numerical processing,

$$
x=\ln \left(1+\frac{n}{P_{i}}\right), \quad n=P_{i}\left(e^{x}-1\right), \quad \mathrm{d} n=P_{i} e^{x} \mathrm{~d} x,
$$

we finally obtain

$$
\begin{aligned}
E\left[N_{i}{ }^{\prime}\right]= & p c_{l, i}+\int_{c_{l, i}}^{\infty} x e^{x} \frac{P_{i}}{\sqrt{2 \pi} \sigma_{n, i}} \\
& \times \exp \left\{-\frac{1}{2}\left[\frac{P_{i}\left(e^{x}-1\right)}{\sigma_{n, i}}\right]^{2}\right\} \mathrm{d} x .
\end{aligned}
$$

Computation of $E\left[{N_{i}}^{\prime}{ }^{2}\right]$ follows the same procedure and yields

$$
\begin{aligned}
E\left[{N_{i}}^{\prime 2}\right]= & p c_{l, i}{ }^{2}+\int_{c_{l, i}}^{\infty} x^{2} e^{x} \frac{P_{i}}{\sqrt{2 \pi} \sigma_{n, i}} \\
& \times \exp \left\{-\frac{1}{2}\left[\frac{P_{i}\left(e^{x}-1\right)}{\sigma_{n, i}}\right]^{2}\right\} \mathrm{d} x .
\end{aligned}
$$

Equations (A5) and (A6), together with Eqs. (22)(25), enable exact computation of the MSE. This requires knowledge of only the measured return power samples $P_{i}$ and the sampling points $R_{i}$ and characterization of the range-dependent systemnoise standard deviation $\sigma_{n, i}$ from system parameters. ${ }^{8}$
Appendix B: Practical Computation of $E\left[N_{i}{ }^{\prime}\right], E\left[N_{i}{ }^{2}\right]$

In nearly all practical cases, the range-dependent SNR associated with each particular sampling cell $R_{i}$ is high enough to enable considerable simplification of Eqs. (A5) and (A6) into the expressions that are presented next. Validity of this approximate method is limited to $\operatorname{SNR}(R) \gg 5$ along all the inversion range with SNR $>9$ being a practical lower bound found by experiment.

For most practical SNR's, ${ }^{7,8}$ the role of the limiting threshold $\nu_{l}$ in Eq. (13) or, equivalently, $n_{l, i}$ is to avoid singularities caused by noise figures that exceed the noise floor $-P_{i}$ rather than clip noise. This is particularly true for any conservative choice of $v_{l}$ well below the expected $S\left(R_{\max }\right)$, as indicated in Subsection 2.B. Under these circumstances, the $n_{l, i}$ threshold is not restrictive, and hence we can write

$$
n_{l, i} \ll-5 \sigma_{n, i} \Rightarrow p \approx 0 .
$$

As a result, the first term of Eq. (A5) can be neglected and $E\left[N_{i}{ }^{\prime}\right]$ can be approximated by

$$
E\left[N_{i}{ }^{\prime}\right] \approx \int_{n_{l, i}}^{\infty} \ln \left(1+\frac{n}{P_{i}}\right) \frac{1}{\sqrt{2 \pi} \sigma_{n, i}} \exp \left[-\frac{1}{2}\left(\frac{n}{\sigma_{n, i}}\right)^{2}\right] \mathrm{d} n,
$$

which, in turn, we can compute by means of a Taylor series expansion by noting that

$$
\ln (1+x)=x-\frac{x^{2}}{2}+\frac{x^{3}}{3}-\frac{x^{4}}{4}+O\left(x^{5}\right), \quad|x| \ll 1,
$$

where $x=n / P_{i}$. Using relation (B1) above, we can rewrite the convergence radius constraint as

$$
\left|\frac{n_{\max }}{P_{i}}\right| \ll 1 \Rightarrow \frac{5 \sigma_{n, i}}{P_{i}} \ll 1 \Rightarrow \mathrm{SNR}_{i} \gg 5,
$$

which indicates that the SNR must be much higher than 5 to ensure series convergence.

Now, if Eq. (B3) is substituted into approximation (B2) and the integration limits are expanded from $-\infty$ to $\infty$, again based on the fact that under the constraint of relation (B1) the contribution of the $\left(-\infty, n_{l, i}\right)$ interval to the integral is negligible, then $E\left[N_{i}{ }^{\prime}\right]$ in approximation (B2) can be expressed in terms of the moments of Gaussian random variable $N_{i}$ as

$$
E\left[N_{i}{ }^{\prime}\right] \approx \frac{E\left[N_{i}\right]}{P_{i}}-\frac{E\left[N_{i}^{2}\right]}{2 P_{i}^{2}}+\frac{E\left[N_{i}^{3}\right]}{3 P_{i}^{3}}-\frac{E\left[N_{i}^{4}\right]}{4 P_{i}^{4}} .
$$

Note that the $n$ th-order moments of a Gaussian random variable $X=N_{i}$ of zero mean and $\sigma$ standard deviation $^{12}$ are

$$
E\left[X^{n}\right]=\left\{\begin{array}{cc}
\frac{\sigma^{n} n !}{2^{n / 2}} & n=\text { even } \\
0 & n=\text { odd }
\end{array}\right.
$$


By substituting these variables into approximation (B5), we finally obtain

$$
E\left[N_{i}{ }^{\prime}\right] \approx-\frac{1}{2} \frac{1}{\mathrm{SNR}_{i}^{2}}-\frac{3}{2} \frac{1}{\mathrm{SNR}_{i}^{4}} .
$$

Derivation of an approximate expression for $E\left[{N_{i}}^{2}\right]$ follows the exact same procedure, and the final result can be expressed as

$$
E\left[N_{i}{ }^{\prime 2}\right] \approx \frac{1}{\mathrm{SNR}_{i}^{2}}+\frac{11}{2} \frac{1}{\mathrm{SNR}_{i}^{4}}+\frac{137}{8} \frac{1}{\mathrm{SNR}_{i}^{6}} .
$$

At this point, it is important to warn that validity of final approximations (B7) and (B8) is conditioned to that of relation (B4), irrespective of the number of terms used for series expansion, because relation (B4) constraint is due to singularitylike behavior of the series for values close to $x=-1$. Cross examination of the exact and the approximate equation sets given by Eqs. (A5) and (A6) and approximations (B7) and (B8), respectively, has shown that the approximate set of approximations (B7)-(B8) can perfectly be used for the computation of the optimum interval length $\left(N_{\text {opt }}\right.$, as described in Subsection 3.B) and its associated MSE [Eqs. (22)-(25) in Subsection 3A], provided that $\operatorname{SNR}\left(N_{\text {opt }}\right)>9$.

We acknowledge the sponsorship of the European Union under the EARLINET (European Aerosol Research Lidar Network to establish an aerosol climatology) contract UE EVR1-CT-1999-40003, the Interministry Committee for Science and Technology (CICYT) under project TIC99-1050-C03-01, and the Interdepartmental Commission for Research and Technological Innovation (CIRIT) of Generalitat de Catalunya under the IMMPACTE project.

\section{References}

1. R. T. H. Collis, "Lidar: a new atmospheric probe," Q. J. R. Meteorol. Soc. 92, 220-230 (1966).

2. R. T. H. Collis and P. B. Russell, "Lidar measurement of particles and gases by elastic backscattering and differential absorption," in Laser Monitoring of the Atmosphere, E. D. Hinkley, ed. (Springer-Verlag, New York, 1976), Chap. 4, pp. 71-102.

3. J. D. Klett, "Stable analytical inversion solution for processing lidar returns," Appl. Opt. 20, 211-220 (1981).
4. G. J. Kunz, "Probing of the atmosphere with lidar," in Proceedings of the Remote Sensing of the Propagation Environment Conference, AGARD-CP-502, (AGARD, Neuilly sur Seine, France, 1992), Vol. 23, pp. 1-11.

5. J. D. Klett, "Lidar calibration and extinction coefficients," Appl. Opt. 22, 514-515 (1983).

6. R. T. Brown, "A new lidar for meteorological application," J. Appl. Meteorol. 12, 698-708 (1973).

7. G. J. Kunz and G. de Leeuw, "Inversion of lidar signals with the slope method," Appl. Opt. 32, 3249-3256 (1993).

8. F. Rocadenbosch, A. Comerón, and D. Pineda, "Assessment of lidar inversion errors for homogeneous atmospheres," Appl. Opt. 37, 2199-2206 (1998).

9. J. J. More, "The Levenberg-Marquardt algorithm: implementation and theory," in Numerical Analysis, Vol. 630 of Springer-Verlag Lecture Notes in Mathematics Series, G. A. Watson, ed. (Springer-Verlag, New York, 1977), pp. 105116.

10. R. Velotta, B. Bartoli, R. Capobianco, L. Fiorani, and N. Spinelli, "Analysis of the receiver response in lidar measurements," Appl. Opt. 37, 6999-7007 (1998).

11. G. J. Kunz, "Effects of detector bandwidth reduction on lidar signal processing," Rep. PHL 1977-31 (Physics and Electronics Laboratory, TNO-FEL, The Hague, 1977).

12. A. Papoulis, Probability, Random Variables and Stochastic Processes (McGraw-Hill, New York, 1991), pp. 345354.

13. A. B. Carlson, "Signal transmission and filtering," in Communication Systems, 3rd ed. (McGraw-Hill, Singapore, 1986), Chap. 3, pp. 177-178.

14. H. Koschmieder, "Theorie der Horizontalen Sichtweite," Beitr. Phys. Freien Atmos. 12, 33-53 (1924).

15. P. W. Kruse, L. D. McGlauchlin, and R. B. McQuiston, Elements of Infrared Technology: Generation, Transmission and Detection (Wiley, New York, 1962).

16. R. J. Barlow, "Least squares," in Statistics: A Guide To The Use Of Statistical Methods In The Physical Sciences, F. Mandl, R. J. Ellison, and D. J. Sandiford, eds. (Wiley, New York, 1989), Chap. 6.

17. W. B. Jones, Introduction to Optical Fiber Communication Systems (Holt, Rinehart \& Winston, New York, 1988), Chaps. 7 and 8.

18. R. M. Measures, Laser Remote Sensing: Fundamentals and Applications (Krieger, Malabar, Fla., 1992), Chap. 4, pp. 138145.

19. R. J. McIntyre, "Multiplication noise in uniform avalanche photodiodes," IEEE Trans. Electron Devices ED-13, 164-168 (1966). 